\title{
Design and Data Analysis of Smart Grid Using Prediction Modelling Technique
}

\author{
K. Bhadraji, M. Lalu, D. Krishna, T. Anil Kumar
}

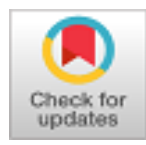

\begin{abstract}
Information analytics and also information scientific research plays a substantial duty in nowadays culture. In the context of Smart Grids (SG), the collection of substantial quantities of information has actually seen the introduction of a myriad of information evaluation strategies. In this paper, we carry out a Systematic Mapping Study focused on obtaining understandings regarding various aspects of SG information evaluation: application sub-domains (e.g., power tons control), elements covered (e.g., projecting), made use of methods (e.g., clustering), tool-support, study approaches (e.g., experiments/simulations), reliability/reproducibility of study. The last objective is to supply a sight of the present standing of study. Projecting of electrical energy need has actually turned into one of one of the most vital locations of study in the electrical power market, as it is an essential element of cost-effective power system monitoring and also preparation. In this context, precise as well as durable tons projecting are meant to play an essential duty in decreasing generation prices, as well as manage the integrity of the power system. Nevertheless, as a result of require heights in the power system, projections are incorrect and also susceptible to high varieties of mistakes. In this paper, our payments consist of a recommended data-mining plan for need modeling with optimal discovery, along with making use of these details to feed the projecting system. For this objective, we have actually taken a various strategy from that of time collection projecting, representing it as a two-stage pattern acknowledgment issue.
\end{abstract}

Index Terms: Smart grid, Data analysis, Forecasts, reliability, efficiency, demand with time, SVM.

\section{INTRODUCTION}

Power is a restricted source which deals with added obstacles as a result of current performance as well as de-carbonization objectives worldwide. The real change in the direction of a lasting, reliable as well as versatile power network calls for an increasing number of complicated approaches for preparation and also running the grid. This recurring makeover leads to enhanced unpredictability and also intricacy in both business purchases as well as in the

Revised Manuscript Received on October 30, 2019

* Correspondence Author

K. Bhadraji, Assistant Professor, Department of EEE, Anurag Group of Institutions, Hyderabad, INDIA.bhadra20723@gmail.com.

M.Lalu, Assistant Professor, Department of EEE, Anurag Group of Institutions, Hyderabad, INDIA.bhadra20723@gmail.com.

D.Krishna, Assistant Professor, Department of EEE, Anurag Group of Institutions, Hyderabad, INDIA.bhadra20723@gmail.com.

Dr.T.Anil Kumar, Assistant Professor, Department of EEE, Anurag Group of Institutions, Hyderabad, INDIA. thalluruanil@gmail.com.

(C) The Authors. Published by Blue Eyes Intelligence Engineering and Sciences Publication (BEIESP). This is an open access article under the CC BY-NC-ND license (http://creativecommons.org/licenses/by-nc-nd/4.0/)

physical circulations of electrical power in the clever grid. This post is concentrated on this last difficulty, as SGs triggered huge quantity of chances in regards to information analytics campaigns: the big schedule of information from the wise framework enables several choice assistances campaigns, however likewise the application of anticipating formulas to boost the offered solutions. Case in points entail power lots projecting forecasting the feasible tons contour that stands for the electrical energy taken in by consumers gradually or Demand Response (DR) standing for tons harmonizing of power supply and also need throughout height hrs[1]. A huge Systematic Mapping Study (SMS) on Smart Grids information analytics, consisting of 267 documents. To our understanding, this is the biggest evaluation in the SG information evaluation domain name in regards to consisted of write-ups[2]. A classification of various SG information evaluation below domain names, with cross-cutting elements such as methods utilized, study approaches, facets explored replicability interest in the accessibility of resource code and also datasets[3]. While an SMS cannot make sure that all research study is covered, it offers an organized tasting device to seek research study facets holistically. Power projections have actually come to be the basis of the clinical choice producing power systems[4]. Based upon the precise forecast of power usage, individuals can successfully lower the price medically allot electrical energy, minimize transmission loss, as well as enhance financial and also social advantages[5]. As a result, the research study on just how to precisely anticipate power intake as well as give effective approaches for circulation of electrical energy is of enormous worth for financial development[6]. Based upon the criteria of assistance support vector machine (SVM). This paper concentrates on exactly how to keep the dimension information and also square away temporary electrical energy intake forecast taking into consideration ecological elements.

\section{RELATED STUDY}

With the fast growth of economic situation, scientific research and also innovations, the range of China's power grid has actually currently placed the very first on the planet[7]. The knowledge of the power grid is primarily based upon the physical basis of the incorporated interaction connect with broadband and also bidirectional fashion, taking on the sophisticated tools, noticing and also determining innovation, as well as progressed regulating and also decision-making systems[8] 
To attain these features, the awareness and also optimization of electrical energy usage forecast has actually ended up being an unpreventable need. Exact power forecast can successfully decrease expenses and also transmission losses, clinically allot electrical energy, enhance financial and also social advantages, and also have a favorable influence on the security of the native environment[9]. Based upon the dispersed storage space, specifically, the HBase data source, the artificial intelligence approach utilizing conventional support vector machine (SVM) is related to research and also educate the ecological Eigen worth information, as well as the created setting design is utilized to examine as well as ultimately anticipated the future ecological information. Lastly, the anticipated outcomes are contrasted as well as evaluated according to the Eigen worth of various ecological aspects[10]. Different Smart Grid ideas have actually been recommended to deal with these obstacles. Significant options are based upon the harmony of typical power system preparation as well as procedure exercise with the most up to date advancements in details as well as interaction modern technology[11]. Nevertheless, a lot of these problems are resolved independently, by various approaches, in various locations of the globe. Now, a widely approved meaning for the "Smart Grid" principle does not exist, and also no typical language to establish this principle.

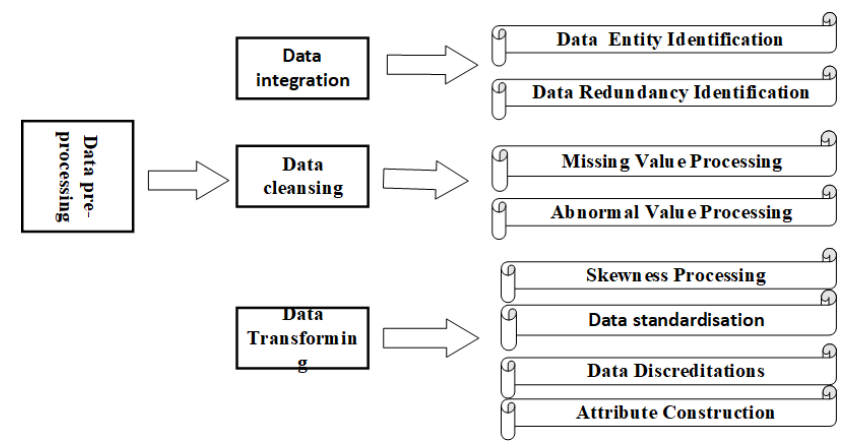

Fig.1. Data Pre-processing Techniques[12].

\section{PROPOSED SYSTEM}

In This paper, SVM category technique is mostly utilized to anticipate as well as assess the electrical power usage. The input vectors are the unbiased ecological aspects associated with the living power are temperature level, adjustment of the variety of individuals, moisture, and also time. The training dataset must be pre-processed prior to educating to enhance the merging as well as computational rate via the equipment finding out procedure. We made use of the stabilized approach in the handling.

$$
x_{i}^{*}=\frac{x_{i}-x_{i}}{\sigma}
$$

In the formula (1) $X_{i}$ are the values of each component of the input vectors, $x_{i}$ are the average values, $\sigma$ are standard deviations. And sigma can get from the formula

$$
\sigma=\sqrt{\frac{\sum_{i=1}^{n}\left(x_{i}-x_{i}\right)^{2}}{n}}
$$

We implemented prediction and comparison through Radial Basis Function based on SVM. RBF is calculated as follows[13]:

$$
\mathrm{K}\left(x_{i}, x\right)=\exp \left[-\frac{\left\|x_{i}-x\right\|}{2 \sigma^{2}}\right]
$$

In SVM, the information as well as linked attributes can be dealt with as a factor as well as vectors in multi-dimensional area. The standard concept of a conventional SVM is to discover an active airplane, which might separate the factors right into different areas. By doing so, we can identify information right into different classifications. In order to reduce the category mistake, the appropriate active aircraft requires to be established. We made use of the SVM to anticipate the temporary electrical energy intake, and also used the primary procedures to assess the outcomes. The initial step is to evaluate the unbiased ecological elements that might influence the temporary electrical power intake. The unbiased ecological variables are taken as the eigenvectors of the artificial intelligence technique and also the actual information is gathered with the sensing units. The acquired information is after that kept in HBase. In the 2nd action, we make the effort, temperature level, moisture, the variety of individuals, as well as various other elements as inputs, with power as outcome. In the 3rd action, the training information collection as well as the examination information collection are both stabilized to quicken the merging of the training network as well as sum up the analytical circulation of the linked examples. In the 4th action, we looked for the ideal specifications making use of SVM feature. In the 5th action, the SVM version is educated according to enhanced criteria, as well as the design is obtained more for assessing as well as forecast projecting. The 6th action, we managed the atmosphere variables for the projecting evaluation, and also contrasted the outcomes, as well as $\mathrm{t}$. The flowchart is displayed in Fig.1

\section{Read the training set and test set}

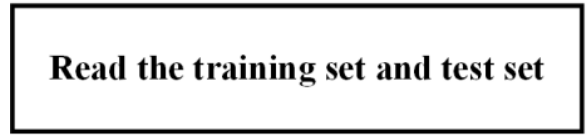

\section{Read the training set and test set}

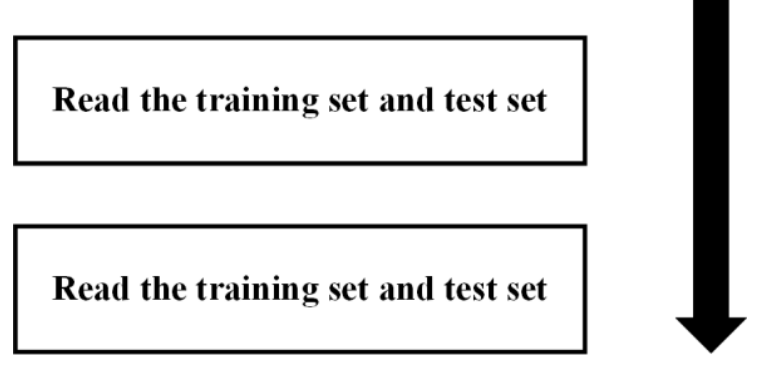

Fig.1. Proposed flow diagram.

The evaluation of the power intake mostly depends upon the existing information in the power grid for information mining and also analytical evaluation. Home electrical energy intake is very connected to several unbiased variables, such as individual's financial degree and also local distinctions. Individual's financial degree has a straight influence on the property electrical power intake. Electrical power intake in locations with a high level of growth is less than that in underdeveloped areas. 
Locations in enjoyable environment have loved one reduced electrical energy usage. The adhering to area offers an in-depth evaluation of ecological aspects based upon speculative outcomes that can impact the quantity of power usage in a solitary area. The moment, variety of individuals, temperature level, as well as moisture is taken as the eigenvalues for artificial intelligence.

\section{SIMULATION RESULTS}

Changes in the everyday lots attributes and also the lots quantity throughout the periods of the year are affected by climate condition consisting of temperature level, moisture, wind, cloud insurance coverage, and also rainfall, along with day size. When it involves regular cycles, these are identified by days, weekend break days, as well as vacations. These several seasonal cycles in the electrical energy usage together with the fad and also non-stationarity in mean and also difference need to be mirrored in the projecting formula in order to develop a durable and also an exact option.

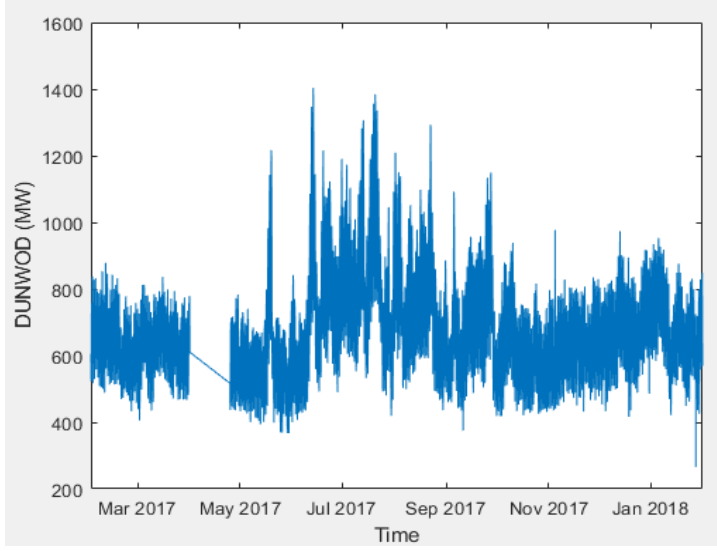

Fig.2. Power with respective of time.

The designs were concentrated on electric power usage optimal discovery in the power system based upon historic information for both electrical energy use and also the climate condition information, consisting of temperature level and also moisture. By doing this, we manage peak discovery as a binary category trouble, unlike a lot of previous research studies where the issue is developed as time collection projecting.

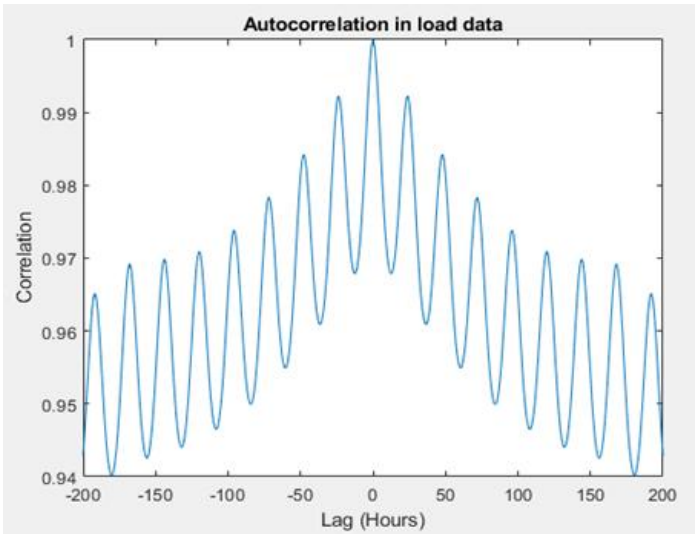

Fig.3. Auto correction in load data

The outcomes reveal that the anticipated power usage making use of 2 ecological variables is dramatically far better than that making use of the solitary ecological variable. The moment and also variety of individuals in the forecast outcomes are one of the most excellent variables, whereas the temperature level and also moisture forecast outcomes are the most awful variables compared to genuine outcomes. Intoxicated of the temperature level as well as moisture.

Box plot represent the power consumption between minimum and maximum levels.

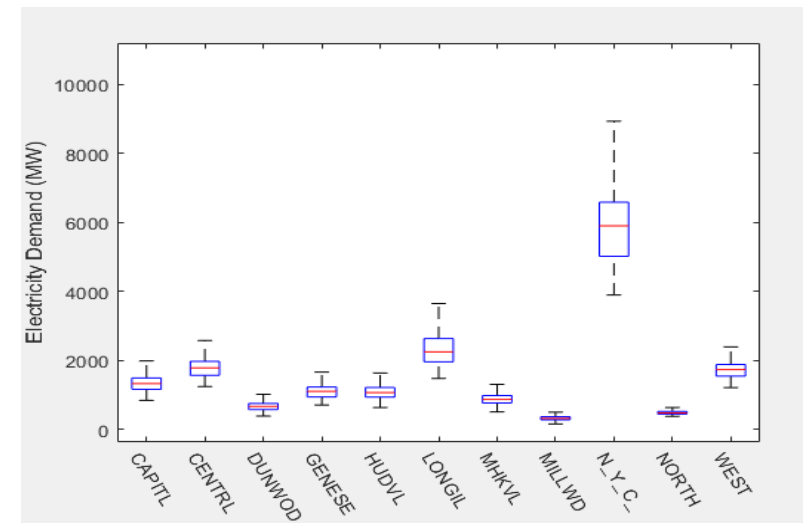

Fig.4. Electricity demand.

With proper scheduling we can reduce the revenue loss. for that we are operating coal new, coal old, Peaker, gas plants. Model development.

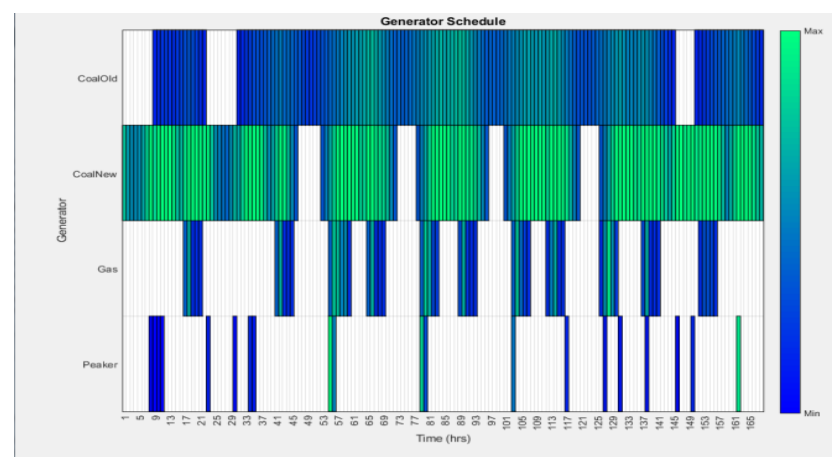

Fig.5.Generator Scheduling

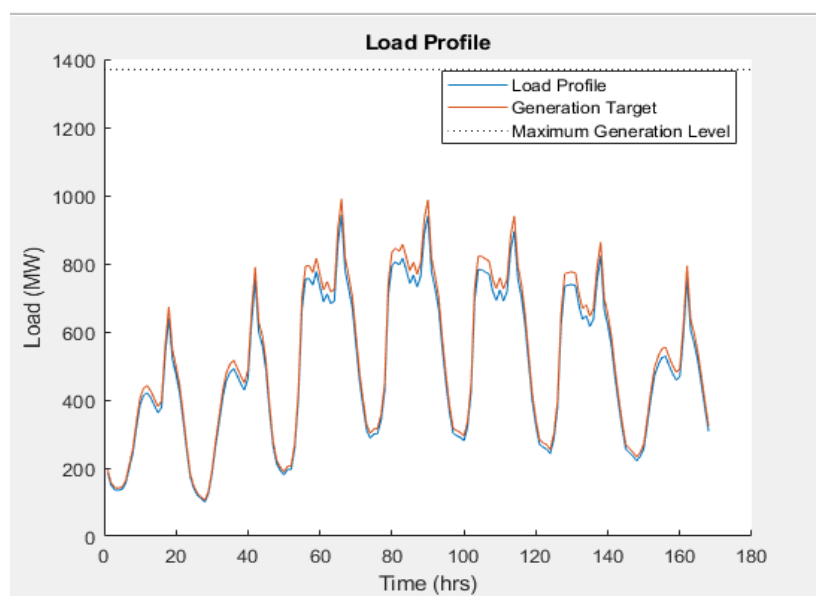

Fig.6. Output results with comparison.

\section{CONCLUSION}

With the enhancing dimension of the power grid, electrical energy forecast has actually progressively shown individuals life.

Published By:

Blue Eyes Intelligence Engineering \& Sciences Publication 
Grid systems have actually been boosted to apply clinical and also efficient power circulation based upon power forecast, to enhance financial efficiency as well as effectiveness of the whole power grid. After extensive information examination as well as evaluation, HBase shows to be remarkable and also is made use of as the information storage space data source. With the evaluation of ecological variables (the moment, variety of individuals, as well as environments) that might have substantial effect on electrical power intake in the short-term, and also the genuine information gotten from the mounted sensing units as well as electronic cameras, we have the ability to carry out temporary power usage forecast. After contrast as well as evaluation of the speculative outcomes of various ecological variables, it shows up that power intake forecast utilizing the moment and also variety of individuals as eigenvalue can create the most effective outcome.

\section{REFERENCES}

1. Haney, A.B., et al., Demand-side management strategies and the residential sector: lessons from international experience. The Future of Electricity Demand: Customers, Citizens and Loads, 2010.

2. Shyam, R., et al., Apache spark a big data analytics platform for smart grid. Procedia Technology, 2015. 21: p. 171-178.

3. Berman, L.E. and Y.G. Rajwan, Data Science Symposium Proceedings. Abstracts for posters presented at the, 2014.

4. Limayem, M., S.G. Hirt, and C.M. Cheung, How habit limits the predictive power of intention: The case of information systems continuance. MIS quarterly, 2007. 31(4).

5. Simmons, S.I., Investigating the impacts of time-of-use electricity rates on lower-income and senior-headed households: A case study of Milton, Ontario (Canada). 2010, University of Waterloo.

6. Dunn, B., H. Kamath, and J.-M. Tarascon, Electrical energy storage for the grid: a battery of choices. Science, 2011. 334(6058): p. 928-935.

7. Dincer, F., The analysis on photovoltaic electricity generation status, potential and policies of the leading countries in solar energy. Renewable and Sustainable Energy Reviews, 2011. 15(1): p. 713-720.

8. Fang, X., et al., Smart grid-The new and improved power grid: A survey. IEEE communications surveys \& tutorials, 2012. 14(4): p. 944-980.

9. Li, J., et al., Big data in product lifecycle management. The International Journal of Advanced Manufacturing Technology, 2015. 81(1-4): p. 667-684.

10. ES, C. and F. FC, SYLLABI OF COMPULSORY FOUNDATION COURSES. CHOICE BASED CREDIT SYSTEM: p. 36.

11. Alkhatib, W., et al. Hybrid Model for Large Scale Forecasting of Power Consumption. in International Work-Conference on Artificial Neural Networks. 2017. Springer.

12. Zhang, Y., T. Huang, and E.F. Bompard, Big data analytics in smart grids: A review. Energy Informatics, 2018. 1(1): p. 8.

13. Chen, H., C.A. Canizares, and A. Singh. ANN-based short-term load forecasting in electricity markets. in 2001 IEEE Power Engineering Society Winter Meeting. Conference Proceedings (Cat. No. 01CH37194). 2001. IEEE. 